\title{
A novel combination regimen of BET and FLT3 inhibition for FLT3-ITD acute myeloid leukemia
}

Haematologica 2021

Volume 106(4):1022-1033

\section{Correspondence:}

MARK LEVIS

levisma@jhmi.edu

Received: January 16, 2020.

Accepted: November 9, 2020.

Pre-published: January 28, 2021.

https://doi.org/10.3324/haematol.2020.247346

(C)2021 Ferrata Storti Foundation

Material published in Haematologica is covered by copyright. All rights are reserved to the Ferrata Storti Foundation. Use of published material is allowed under the following terms and conditions:

https://creativecommons.org/licenses/by-nc/4.0/legalcode. Copies of published material are allowed for personal or internal use. Sharing published material for non-commercial purposes is subject to the following conditions:

https://creativecommons.org/licenses/by-nc/4.0/legalcode, sect. 3. Reproducing and sharing published material for commercial purposes is not allowed without permission in writing from the publisher.

\author{
Lauren Y. Lee, ${ }^{1}$ Yoshiyuki Hizukuri, ${ }^{2}$ Paul Severson, ${ }^{3}$ Benjamin Powell, ${ }^{3}$ \\ Chao Zhang, ${ }^{3}$ Yan Ma, ${ }^{3}$ Maiko Narahara, ${ }^{2}$ Hiroyuki Sumi, ${ }^{2}$ \\ Daniela Hernandez, ${ }^{1}$ Trivikram Rajkhowa, ${ }^{1}$ Gideon Bollag ${ }^{3}$ and Mark Levis ${ }^{1}$
}

${ }^{1}$ Sidney Kimmel Comprehensive Cancer Center, Johns Hopkins University, Baltimore, MD, USA; ${ }^{2}$ Daiichi Sankyo Co. Ltd., Tokyo, Japan and ${ }^{3}$ Plexxikon Inc., Berkeley, CA, USA

\section{ABSTRACT}

A cute myeloid leukemia (AML) patients with FLT3-ITD mutations have a high risk of relapse and death. FLT3 tyrosine kinase inhibitors improve overall survival, but their efficacy is limited and most patients who relapse will ultimately die of the disease. Even with potent FLT3 inhibition, the disease persists within the bone marrow (BM) microenvironment, mainly due to BM stroma activating parallel signaling pathways that maintain pro-survival factors. BET inhibitors suppress pro-survival factors such as MYC and BCL2, but these drugs thus far have shown only limited single-agent clinical potential. We demonstrate here, using pre-clinical and clinical correlative studies, that the novel 4-azaindole derivative, PLX51107, has BET-inhibitory activity in vitro and in vivo. The combination of BET and FLT3 inhibition induces a synergistic anti-leukemic effect in a murine xenograft model of FLT3ITD AML, and against primary FLT3-ITD AML cells co-cultured with BM stroma. Using suppression of MYC as a surrogate for BET inhibition, we demonstrate BET inhibition in human patients. The short plasma half-life of PLX51107 results in intermittent target inhibition to promote tolerability while overcoming the protective effect of the microenvironment. Mechanistically, the synergistic cytotoxicity is associated with suppression of key survival genes such as MYC. These data provide the scientific rationale for a clinical trial of a BET plus FLT3 inhibitor for the treatment of relapsed/refractory FLT3-ITD AML. A clinical trial of PLX51107 as monotherapy in patients with different malignancies is underway and will be reported separately.

\section{Introduction}

Internal tandem duplication (ITD) mutations of the receptor tyrosine kinase FLT3 are associated with a poor prognosis in acute myeloid leukemia (AML), both at diagnosis and in the relapsed/refractory setting. ${ }^{1-3}$ Several FLT3 tyrosine kinase inhibitors (TKI) have recently been approved for use in this disease subtype at various stages of the disease, and outcomes for patients with FLT3-mutated AML are now improving. ${ }^{4}$ Unfortunately, relapses still occur, and the presence of a FLT3ITD mutation is one of the worst prognostic factors at relapse, with few patients achieving cure. ${ }^{5,6}$ FLT3 inhibitors such as gilteritinib and quizartinib only prolong survival and very few relapsed patients are cured. ${ }^{7,8}$ In relapsed FLT3-ITD AML patients treated with these drugs, circulating peripheral blasts are eliminated, but marrow blasts persist, ${ }^{9,10}$ and resistance-conferring FLT3 point mutations or RASpathway mutations soon emerge..$^{11-13}$ Combinations with chemotherapy and hypomethylating agents are under investigation, but a combination with a more targeted agent might represent a better option.

We and others have previously reported that marrow blasts are protected from the FLT3 inhibition via signaling through stromal-derived cytokines., ${ }^{9,10,14,15}$ Rather than target individual cytokines, we looked further downstream at the convergence of their signaling pathways, which drive transcription of pro-survival genes such as MYC. This led us to explore the inhibition of bromodomain and extra-terminal domain (BET) proteins, which are master transcription regulators. ${ }^{16}$ This family of proteins includes $\mathrm{BRD} 2, \mathrm{BRD} 3, \mathrm{BRD} 4$ and $\mathrm{BRDT}$. They associate with acetylated 
chromatin to control transcriptional activation and cell cycle progression in leukemogenesis. ${ }^{17}$ Of the four BET family members, BRD4 has been of particular interest in drug development as it directly recruits positive transcription elongation factor complex $\mathrm{b}(\mathrm{P}-\mathrm{TEFb})$ to promote transcription of MYC and other proto-oncogenes. ${ }^{18}$

Previous work revealed that the combination of BET inhibition and FLT3 inhibition led to synergistic cytotoxicity in FLT3-ITD AML cells. ${ }^{19}$ Although this was an important proof-of-principle, these studies were carried out using cells in suspension culture, which does not address the fundamental problem of microenvironmentmediated resistance to FLT3 inhibition. Furthermore, in order to translate this in vitro combination appropriately into the clinic, a BET inhibitor is needed that can achieve adequate MYC suppression in humans. We report here that the combination of PLX51107, ${ }^{20}$ a structurally novel BET inhibitor currently in early phase clinical studies, and quizartinib, a highly selective FLT3 inhibitor, results in synergistic cytotoxic effects in FLT3-ITD AML blasts on bone marrow (BM) stroma, and that this combination represents a clinically viable strategy to overcome microenvironment-mediated resistance to FLT3 inhibition.

\section{Methods}

\section{FLT3 and BET inhibitors}

PLX51107 was synthesized at Plexxikon Inc. (Berkeley, CA, USA), a Daiichi Sankyo subsidiary. Quizartinib was obtained from LC Laboratories (Woburn, MA, USA). Stock solutions in dimethyl sulfoxide (DMSO) were stored at $-20^{\circ} \mathrm{C}$. Dilutions from the stock solutions were prepared in RPMI (Gibco, Waltham, MA, USA) with 10\% fetal bovine serum (FBS) (Gemini Bio Products, Sacramento, CA, USA), penicillin/streptomycin, and $2 \mathrm{M} \mathrm{L}$-glutamine (Gibco, Waltham, MA, USA). The final concentration of DMSO in all experiments was $\leq 0.1 \%$.

\section{Patients' samples}

Patients' leukemia samples, as well as plasma and BM from healthy donors, were acquired under an institutional review board-approved protocol from the Johns Hopkins Tumor and Cell Procurement Bank. Patients gave informed consent according to the Declaration of Helsinki.

\section{Cell cultures}

Cell lines and primary leukemia cells were cultured in RPMI with $10 \% \mathrm{FBS}$, penicillin/streptomycin, and L-glutamine at $37^{\circ} \mathrm{C}$ in $5 \% \mathrm{CO}_{2}$ OCI-AML3 (expressing a wild-type FLT3 gene, an NPM1 gene mutation [type A], and a DNMT3A R882C mutation) and Molm14 cells (expressing a 21 bp FLT3-ITD mutation) were purchased from Deutsche Sammlung von Mikroorganismen and Zellkulturen, Braunschweig, Germany. MV4-11 cells (FLT3-ITD mutated) were from American Type Culture Collection, Manassas, VA, USA.

\section{Cytotoxicity assays}

Primary co-cultures in 96-well plates were incubated with specified drug treatments for 72 hours (h) prior to the assessment of cytotoxicity by a dimethyl-thiazole diphenol tetrazolium bromide (MTT) assay (Roche Diagnostics, Indianapolis, IN, USA) as described. ${ }^{21}$ Experimental duplicates were also concurrently counted for cell viability by trypan blue exclusion under light microscopy.

\section{Immunoblotting}

The antibodies to probe for MYC and $\beta$-actin were obtained from Cell Signaling Technologies (\#5605, Danvers, MA, USA) and $\beta$-actin (13E5, Danvers, MA, USA).

\section{Plasma inhibitory activity assays}

Plasma for the plasma inhibitory activity (PIA) assays was collected from patients enrolled in a phase Ib/IIa doseescalation/expansion study of PLX51107 (clinicaltrials.gov identifier: NCT02683395). Plasma samples were collected from cycle 1 on day (d) 1 at $0,0.5,1,2,3,5,7$, and $9 \mathrm{~h}$, and pre-dose on $\mathrm{d} 2$ (i.e., 24 $h$ post $\mathrm{d} 1$ dose). The samples were stored at $-80^{\circ} \mathrm{C}$ and used within 12 months of collection for the PIA assays.

The PIA assay was adapted from a previously described approach. ${ }^{22}$ OCI-AML3 cells were incubated with each plasma sample for $3 \mathrm{~h}$ at $37^{\circ} \mathrm{C}$ in microcentrifuge tubes. Each OCI-AML3 sample was then centrifuged and washed a total of five times each in phosphate buffered saline (PBS) prior to cell lysis. Lysates were cleared by centrifugation. $50 \mu \mathrm{g}$ of lysates were subjected to sodium dodecyl sulphate-polyacrylamide gel electrophoresis (SDSPAGE) and immunoblot, as described above. Membranes were probed for antibodies against MYC and $\beta$-actin, as described above.

\section{Colony-forming assays}

Colony-forming assays of normal human BM progenitors were performed as described. ${ }^{23}$

Information concerning primary stromal culture and leukemic cell co-culture, xenograft studies, RNA sequencing and quantitative-polymerase chain reaction (PCR) is available in the Online Supplementary Methods.

\section{Results}

Previous work demonstrated a synergistic cytotoxic effect of the BET inhibitor JQ1 and FLT3 inhibition in FLT3-ITD AML cells in suspension culture. ${ }^{19}$ To pursue this concept in vivo, we first tested it in a mouse model of FLT3-ITD AML. JQ1 is poorly suited to in vivo application. However, PLX51107 is a structurally distinct BET inhibitor that exhibits low nanomolar affinity and a unique binding position, ${ }^{20}$ and is rationally designed for therapeutic use in humans. PLX51107, which has no activity as a FLT3 inhibitor (Online Supplementary Figure S1), potently inhibited the in vitro growth of two AML cell lines harboring FLT3 ITD mutations, MV4-11 and MOLM-14, with $\mathrm{IC}_{50}$ values of 62 and $79 \mathrm{nM}$, respectively (Online Supplementary Figure S2), suggesting that these cell lines are susceptible to BET inhibition. Despite its short plasma half-life, oral administration of PLX51107 led to significant transcriptional changes of a BET target gene HEXIM1 in MV4-11 cells xenografted in female SCID mice, confirming target engagement in vivo. ${ }^{20}$ Here we used the same model but extended the duration of the study to examine the in vivoefficacy of PLX51107 as a single agent or in combination with quizartinib in treating FLT3-ITD AML.

We first evaluated three oral doses $(10,20$ and $40 \mathrm{mg} / \mathrm{kg}$ daily) of PLX51107 for efficacy in blocking MV4-11 xenograft growth. PLX51107 treatments demonstrated dose-dependent suppression of tumor growth (Figure 1A), achieving $>50 \%$ tumor growth inhibition at $10 \mathrm{mg} / \mathrm{kg}$ dose (corresponding $\mathrm{AUC}_{0-24}=29,300 \mathrm{ng} \times \mathrm{h} / \mathrm{mL}$ ) and above. PLX51107 dosed at $40 \mathrm{mg} / \mathrm{kg}$ resulted in tumor regression between $\mathrm{d} 4$ and $\mathrm{d} 13$ of dosing. By $\mathrm{d} 14$ (the last 
A Treatment $\begin{array}{ll}-0-\text { Vehicle } & -0-\text { PLX51107 20mg/kg } \\ -\triangle \text { PLX51107 10mg/kg } & -\square-P L X 5110740 \mathrm{mg} / \mathrm{kg}\end{array}$

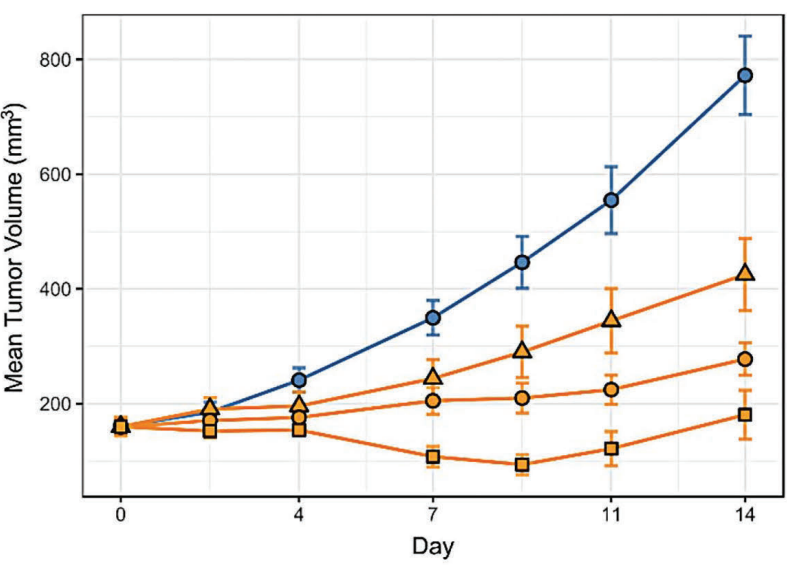

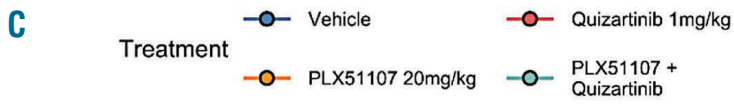

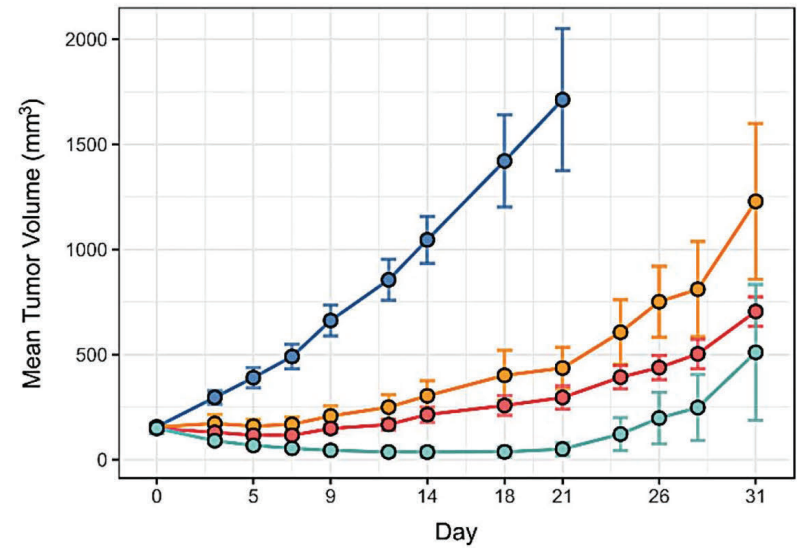

E

$$
\begin{aligned}
& \text { Treatment }-0 \text { - Vehicle -O- Quizartinib } 5 \mathrm{mg} / \mathrm{kg} \\
& \text {-O- PLX51107 20mg/kg -O- PLX51107+ }
\end{aligned}
$$

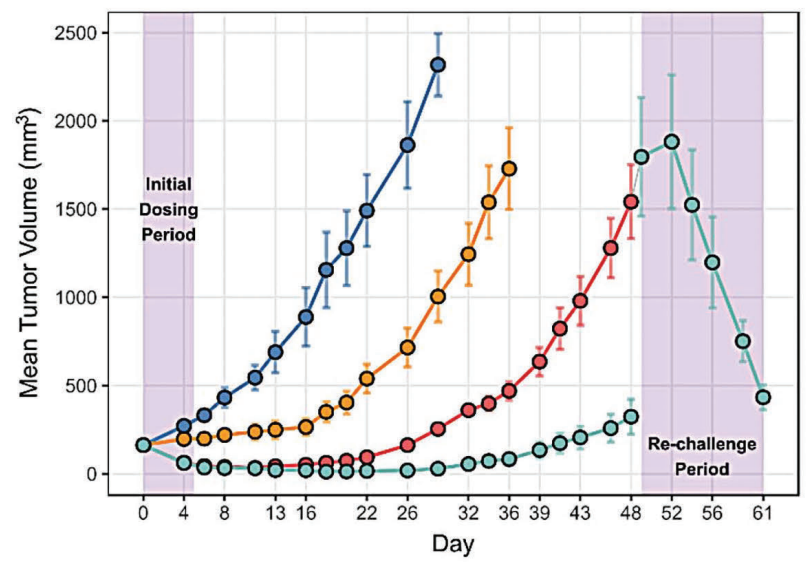

B

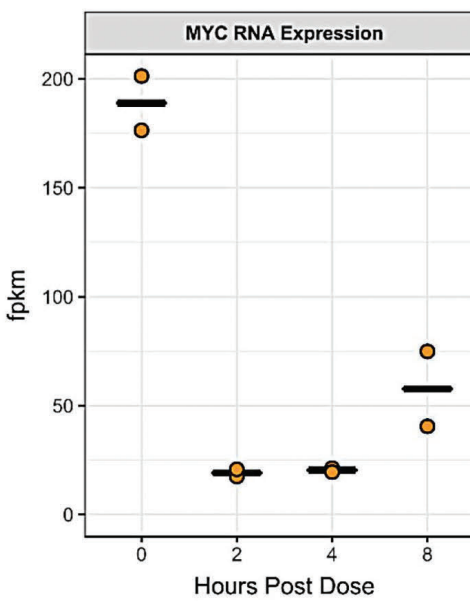

Treatment

- PLX51107 20mg/kg
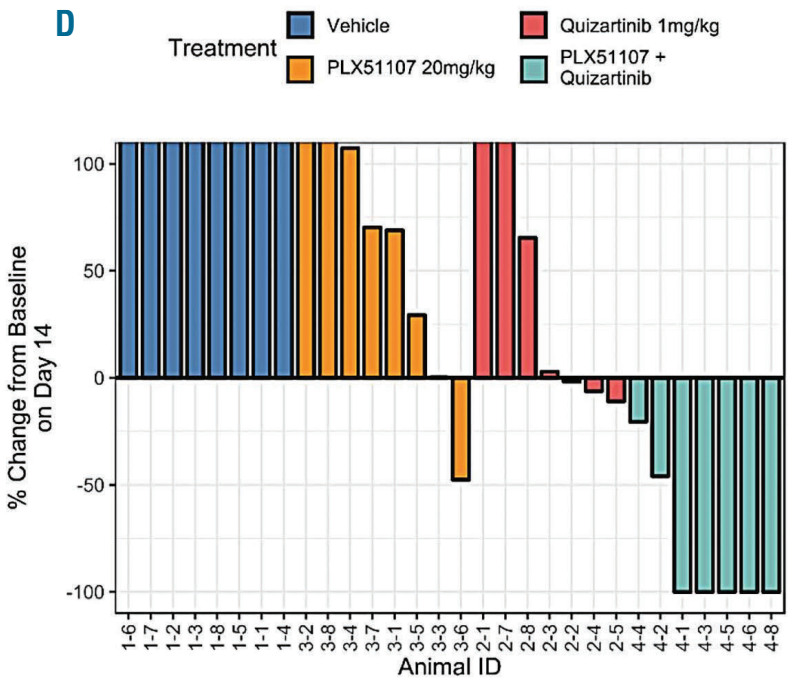

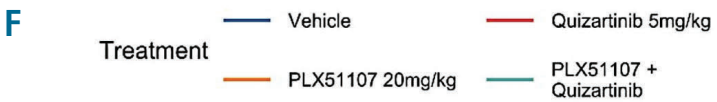

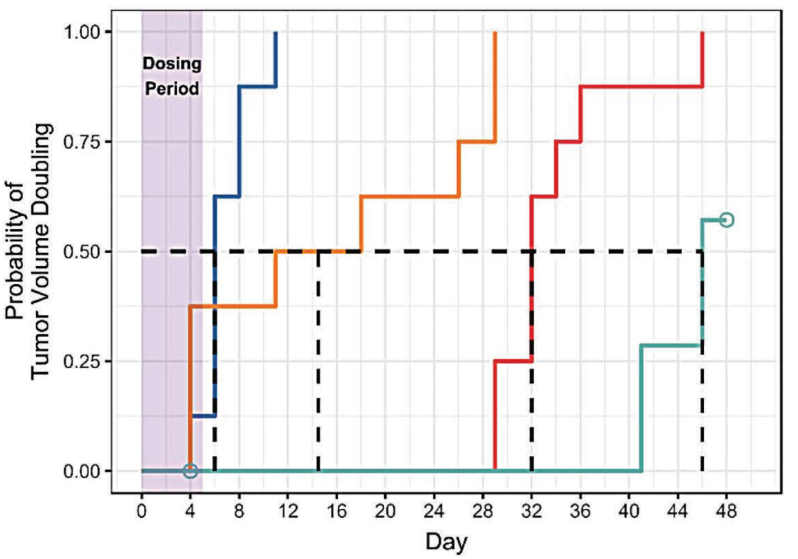

Figure 1. In vivo efficacy of PLX51107 and quizartinib in MV4-11 xenograft models. (A) Dose-response relationship of PLX51107 single agent activity after 14-day treatment; (B) MYC gene expression measured pre-dose, 2, 4 and 8 hours after a single $20 \mathrm{mg} / \mathrm{kg}$ dose of PLX51107. Two mice were taken down at each time point for the gene expression profiling. (C and D) $20 \mathrm{mg} / \mathrm{kg}$ PLX51107 were combined with low-dose (1 mg/kg) quizartinib in a continuous (25-day) dosing regimen. Treatment of MV4-11 xenografts with quizartinib and/or PLX51107 results in tumor control, particularly in the combination arm. (D) 5 of 7 mice treated with the combination had no measurable tumor on day 14. (E and F) $20 \mathrm{mg} / \mathrm{kg}$ PLX51107 was combined with high-dose (5 mg/kg) quizartinib for 5 days. On day 49 , following regrowth of tumors in the short-term single-agent quizartinib arm, treatment was restarted with 20 mg/kg PLX51107 plus 5 mg/kg quizartinib to monitor further tumor control. 
day of dosing and tumor measurement), PLX51107 treatment groups showed $57 \%, 81 \%$, and $97 \%$ tumor growth inhibition (\%TGI) at 10,20, and $40 \mathrm{mg} / \mathrm{kg}$ doses, respectively. These results indicate that PLX51107 has single agent activity against AML in vivo. No adverse effects were observed at doses of 10 and $20 \mathrm{mg} / \mathrm{kg}$. The high dose (40 $\mathrm{mg} / \mathrm{kg}$ ) was also tolerated but exhibited some toxicity (e.g., body weight loss in some animals) which recovered over time. The toxicity is likely due to the high exposure of PLX51107 at this dose $\left(\mathrm{AUC}_{0-24}=211,000 \mathrm{ng} \times \mathrm{h} / \mathrm{mL}\right)$. Based on in-life tolerability and body weight changes, the maximum dose without body weight loss in this study was $20 \mathrm{mg} / \mathrm{kg}$ with corresponding $\mathrm{AUC}_{0-24}$ of 78,800 $\mathrm{ng} \mathrm{x}$ $\mathrm{h} / \mathrm{mL}$. In the same model, we tested whether the PLX51107 treatment had any effect of MYC gene expression. Mice were given a single dose of $20 \mathrm{mg} / \mathrm{kg}$ PLX51107 and MYC RNA was measured in the tumor tissues after 2, 4 and $8 \mathrm{~h}$ (Figure 1B). Relative to pre-dose MYC expression, we observed a $90 \%$ decrease in MYC gene expression between 2 and $4 \mathrm{~h}$ post dose that only partially rebounded at $8 \mathrm{~h}$ post dose.

We next evaluated efficacy of the quizartinib-PLX51107 combination in the MV4-11 xenograft model using two different dosing regimens (PLX51107 at $20 \mathrm{mg} / \mathrm{kg}$ was used in both cases). The first experiment examined the effect of PLX51107 in combination with continuous (25 days), low-dose (1 mg/kg) quizartinib (Figure 1C). d0 is first day of dosing. On d5, PLX51107 alone almost completely suppressed tumor growth (TGI=98\%) while quizartinib monotherapy and quizartinib-PLX51107 combination caused tumor regression $(22 \%$ and $55 \%$, respectively). Tumors in mice in the single agent quizartinib group progressed on $\mathrm{d} 9$ whereas mice in the combination group had maintained tumor regression until d26. On d14, 5 out of 7 animals in the quizartinib-PLX51107 group had no measurable tumors (100\% decrease) (Figure 1D).

The third MV4-11 xenograft study examined the effect of PLX51107 on the duration of response after short-term (5 days) co-administration with high-dose $(5 \mathrm{mg} / \mathrm{kg}$ ) quizartinib (Figure 1E and F). By d4, PLX51107 alone delayed tumor growth (\%TGI=68\%) while quizartinib monotherapy and quizartinib-PLX51107 combination caused significant tumor regression $(61 \%$ and $62 \%$, respectively). The benefit of adding PLX51107 became evident when the durations of response were compared. Mice in the $5 \mathrm{mg} / \mathrm{kg}$ quizartinib group progressed on d26 (22 days after the last quizartinib dose) whereas mice in the quizartinib-PLX51107 group maintained tumor regression until d39. Two out of eight animals in the combination group achieved complete remission (CR) by $\mathrm{d} 4$ and six achieved $\mathrm{CR}$ by d18, while no animal in the single agent groups experienced a CR. Furthermore, tumors in mice that relapsed after short-term, single-agent quizartinib treatment decreased in size when challenged with quizartinib + PLX51107 on d49.

Taken together, these studies showed that, in a mouse xenograft model, PLX51107 enhanced the response of AML cells to continuous, low-dose quizartinib treatment and significantly improved the duration of response to short-term, high-dose quizartinib treatment without attendant toxicity. In addition, PLX51107 showed activity against AML not controlled by prior quizartinib treatment.

The efficacy observed in the xenograft studies reflects primarily the synergy between two cytotoxic agents acting at different levels of oncogenic signaling (i.e., proximal and downstream) in malignant cells not associated with BM stroma. In the clinical setting, BET inhibition could reduce the level of survival factors such as MYC in blasts on stromal cells, thereby undermining the resistance to the effects of FLT3 inhibition conferred by the microenvironment. Before we could test this hypothesis using co-culture of primary blast samples with mesenchymal stromal cells, it was necessary to determine the concentration of PLX51107 in culture medium that would give rise to a pharmacodynamic effect comparable to that observed in humans at clinically achievable drug exposure. To that end, we developed a surrogate assay modeled on the one we had previously used to estimate FLT3 inhibition in vivo. ${ }^{22}$ A phase I dose escalation study of PLX51107 in patients with solid tumors and hematologic malignancy (clinicaltrials.gov identifier: NCT02683395) is underway, and the results of this trial will be published separately. Plasma samples collected from patients on this study were incubated with OCI-AML3 cells (NPM1-mutated, FLT3wild-type). The degree and duration of MYC inhibition were quantified by immunoblotting and densitometry. We refer to this value as the plasma inhibitory activity for MYC (or MYC PIA). The OCI-AML3 cell line was selected because MYC expression is suppressed by PLX51107 (Figure 2A and B) but not by quizartinib (Figure 2C). Such specificity makes it possible to use the developed assay to measure the BET-mediated (FLT3-independent) effect in a quizartinib-PLX51107 combination trial.

Six dose levels of PLX51107 were evaluated in cancer patients. The MYC PIA assay showed that suppression of MYC appeared at $30 \mathrm{mg} /$ day and peaked at $120 \mathrm{mg} /$ day (Figure 3A). A further increase in dose to $160 \mathrm{mg} /$ day did not lead to enhanced MYC suppression. For each plasma sample that was used to assay for MYC PIA, the plasma concentration of PLX51107 within that sample was measured. When the MYC PIA results are plotted against concentrations of the drug, the points align with the standard curve (see Figure 2B) of PLX51107 in plasma (Figure 3B). We also plotted all MYC PIA values from the $120 \mathrm{mg} /$ day cohort (Figure 3C), from which we estimate the duration of MYC inhibition to be approximately $6 \mathrm{~h}$ at this dose. The mean plasma concentration over the first $6 \mathrm{~h}$ is $3.3 \mu \mathrm{M}$, which achieves approximately $80 \%$ inhibition of MYC in the PIA. An equivalent effect is achieved in regular culture medium by PLX51107 at a concentration of $250 \mathrm{nM}$.

Even potent FLT3 inhibitors such as gilteritinib and quizartinib are unable to eradicate FLT3-ITD AML blasts on BM stroma as single agents (Figure 4A), which we and others have observed previously. ${ }^{9,10,14,15}$ Having established that PLX51107 could achieve target inhibition and tumor control in vivo, we next asked if stromal-mediated resistance to FLT3 inhibition in vitro could be overcome with the addition of BET inhibition. In previous studies, the concentration of quizartinib necessary to achieve complete FLT3 inhibition in blasts on BM stroma was established to be $50 \mathrm{nM}$ (in culture medium) ${ }^{24,25}$ From the above experiments, the plasma concentration of PLX51107 in patients (Figure 3B) that resulted in MYC inhibition can be achieved by 250 nM PLX51107 in cell culture medium (Figure 2A). Therefore, primary blast samples from patients with FLT3-ITD AML were co-cultured with mesenchymal stromal cells obtained from healthy marrow donors in the presence of $50 \mathrm{nM}$ quizartinib, $250 \mathrm{nM}$ PLX51107, or the combination, for $72 \mathrm{~h}$, and then the cells were assayed for viability compared with untreated con- 
trol (Figure 4B). There was inter-sample variation in the cytotoxic response to quizartinib (consistent with that previously observed; see Figure 4A), while PLX51107 by itself induced minimal cytotoxicity. The drugs induced no apparent effect on the proliferation of the stromal cells, either alone or in combination (data not shown). Importantly, the combination of the two drugs resulted in a synergistic cytotoxic effect against the patient-derived blasts (Figure 4B). We used ten samples in this fashion (the clinical data for these ten patients are detailed in Online Supplementary Table S1) and averaged the responses in an MTT assay to demonstrate a consistent synergy by median effect analysis (Figure 4C). We confirmed these results by performing direct cell counts using Trypan Blue exclusion to identify viable cells (data not shown). However, this synergistic effect was the result of continuous exposure to

A

PLX51107 Media Dose Response

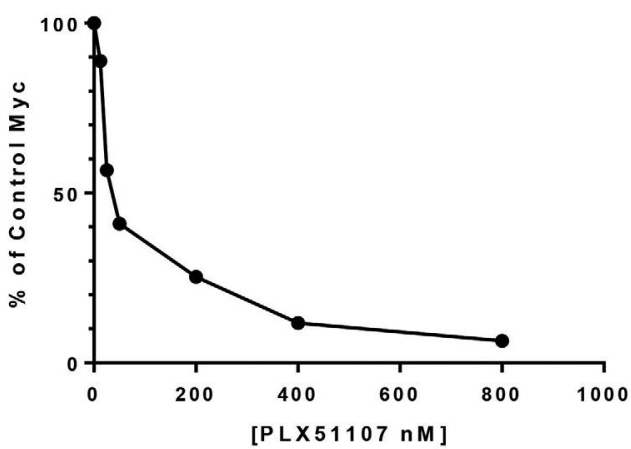

c-Myc

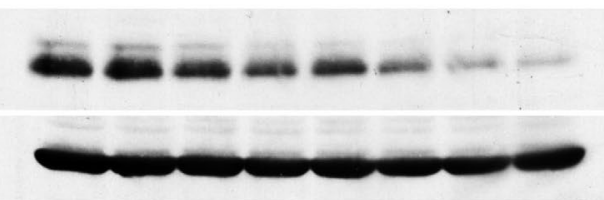

Actin

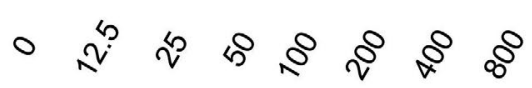

PLX51107(nM)

C

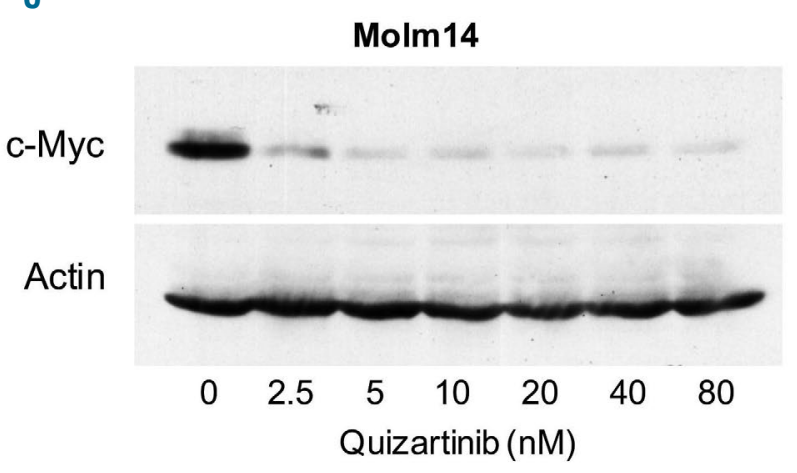

BET inhibition. The PIA data (see Figure 3) indicated that BET inhibition only lasted for approximately 6 h per day in patients. Therefore, we modeled this intermittent BET inhibition by incubating the blasts with PLX51107 for $6 \mathrm{~h}$ per day during the 3-day exposure period, with quizartinib continuously present (Figure 4D). Interestingly, the degree of cytotoxicity with intermittent exposure to BET inhibition was similar to that observed with continuous exposure. The explanation for this may be that the effects of PLX51107 on transcription persist for several hours after exposure to the drug, at least as seen in the mouse xenograft studies (Figure 1) and as observed by others. ${ }^{20}$ We repeated the combination experiment using a different FLT3 inhibitor, gilteritinib, to confirm that the synergy was due to a FLT3 TKI class effect (Online Supplementary Figure S3).
B

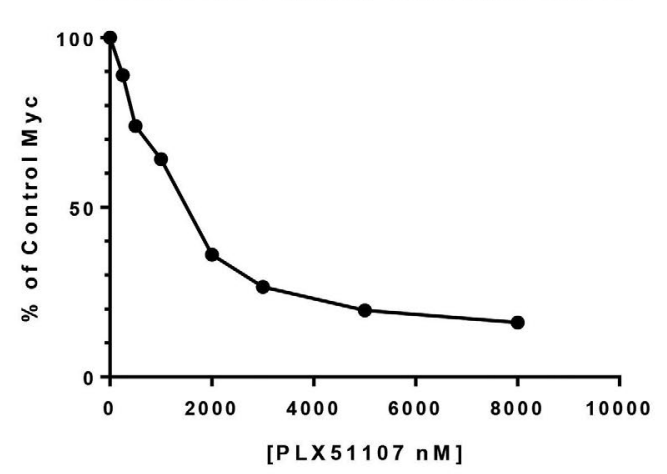

c- Myc

Actin

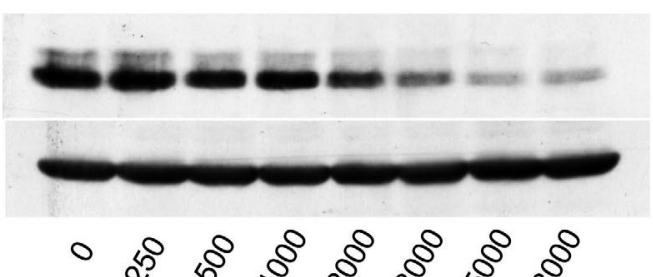

PLX51107(nM)

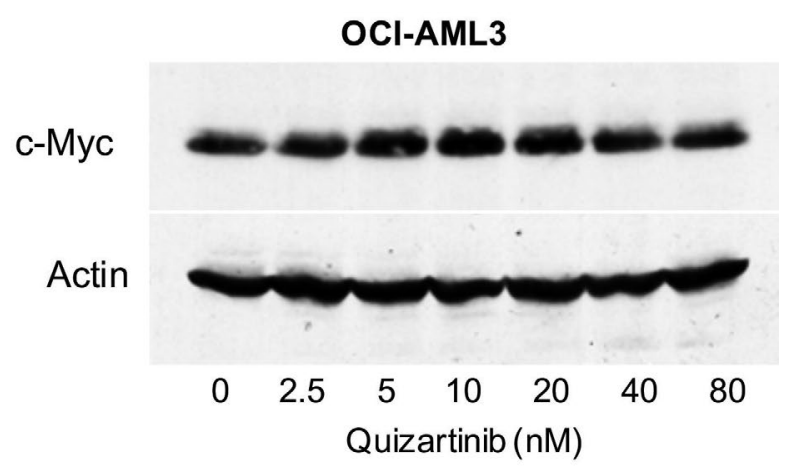

Figure 2. In vitro and in vivo correlative analysis of PLX51107 pharmacodynamic effect. OCl-AML3 cells were treated with increasing concentrations of PLX51107 for 3 hours (h) in either (A) RPMI/10\% fetal bovine serum media or (B) control human plasma. Cells were then lysed and probed for MYC by immunoblotting. The quantitative analyses were performed by densitometry. The dose response curves in the graphs were generated from the average densitometry values of three separate experiments. Representative blots from both media and plasma conditions are shown. (C) Molm14 (FLT-ITD FLT3 receptor) and OCl-AML3 (wild-type FLT3 receptor) cells were treated with quizartinib for $3 \mathrm{~h}$. Cells were then lysed and analyzed for MYC protein expression by immunoblotting, as described in the Methods section. 
We repeated the combination treatments using three different primary AML samples on stroma and analyzed gene expression patterns at different time points using RNA sequencing. Using a false discovery rate (FDR) cutoff of 0.05 in transcript level, global changes in gene expression were minimal with quizartinib at $6 \mathrm{~h}$ and modest at $24 \mathrm{~h}$ (Figure 5A). Not surprisingly, the addition of PLX51107 resulted in more dramatic changes in gene expression at both $6 \mathrm{~h}$ and $24 \mathrm{~h}$. The effects of BET inhibition on gene expression were transient, as a 6-h (e.g.,
A

\section{Cohort 3-60 mg \\ Subject 11}

C1D2

Timepoint (h) Pre $\begin{array}{lllllllll}0.5 & 1 & 2 & 3 & 5 & 7 & 9 & \text { Pre }\end{array}$

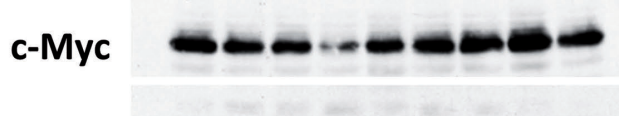

Actin

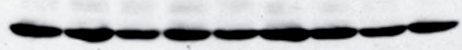

C1D1

Cohort 4-90 mg

Subject 15

C1D2

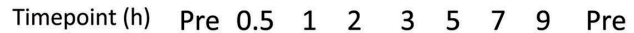

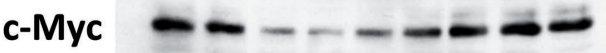

Actin

B

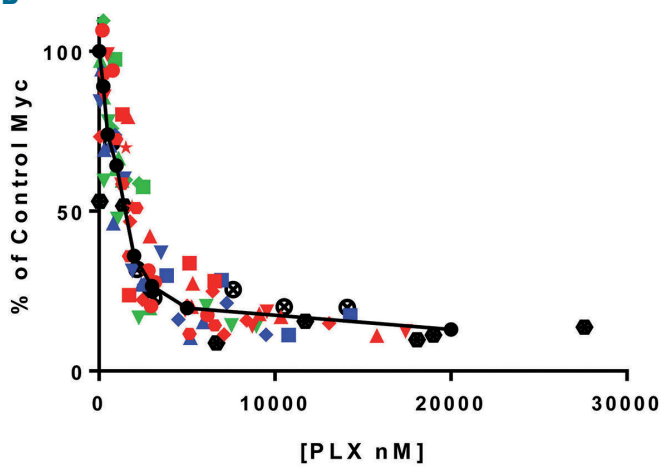

C

Cohort 5

$120 \mathrm{mg} P L X 51107$

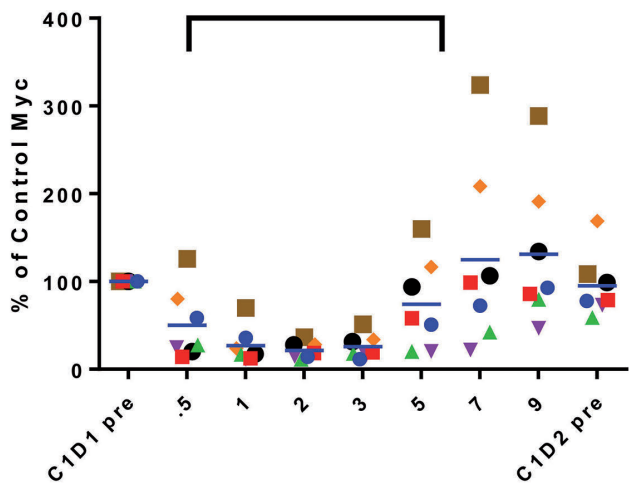

Timepoints $(h)$

\section{Cohort 5-120 mg \\ Subject 23 \\ C1D2}

Timepoint (h) Pre $\begin{array}{lllllllll}0.5 & 1 & 2 & 3 & 5 & 7 & 9 & \text { Pre }\end{array}$

c-Myc - - - - - -

Actin

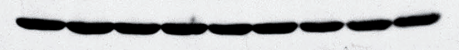

Cohort 6-160 mg

Subject $29 \quad$ C1D2

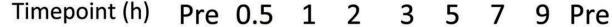

c-Myc

Actin

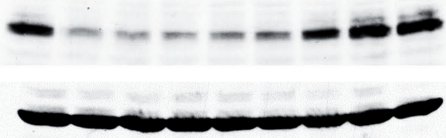

$\rightarrow$ Standard

- Sub 10

A Sub 11

V Sub 12

- Sub 13

- Sub 14

- Sub 15

$\checkmark$ Sub 16

- Sub 17

- Sub 19

$\checkmark$ Sub 22

A Sub 23

- Sub 24

- Sub 25

- Sub 26

* Sub 27

(-) Sub 29

- Sub 31
Cohort 3

$60 \mathrm{mg}$

Cohort 4

$90 \mathrm{~m} \mathrm{~g}$

Cohort 5

$120 \mathrm{~m} \mathrm{~g}$

Cohort 6

$160 \mathrm{mg}$
Sub 19

- Sub 22

A Sub 23

v Sub 24

- Sub 25

- Sub 26

- Sub 27

Figure 3. Plasma inhibitory activity (PIA) assays for MYC. (A) PIA assays for MYC were performed on patients' plasma samples collected at different time points throughout cycle 1 day 1 in a dose escalation study of PLX51107. Out of six cohorts, only the samples from the four cohorts receiving the highest doses exhibited MYC suppression in the MYC surrogate assay (shown here). Cohorts 5 and 6, receiving $120 \mathrm{mg}$ or $160 \mathrm{mg}$ of PLX51107 per day, produced the maximal MYC suppressive effect, which lasted for approximately 6 hours (h). (B) A total of 17 patients' plasma sets were analyzed by the PIA assay. Each symbol represents a time point for a particular patient. The MYC PIA result for each point is plotted against the concentration of PLX51107 measured in that sample. The plasma standard curve (solid black line) was generated from OCI-AML3 dose responses in Figure 1A. (C) PIA assays for cohort 5 are plotted against time after dosing. Values for MYC expression were obtained through densitometry and the 6-h length of MYC suppression is indicated by the bracket. 
A

100 n M Gilteritinib

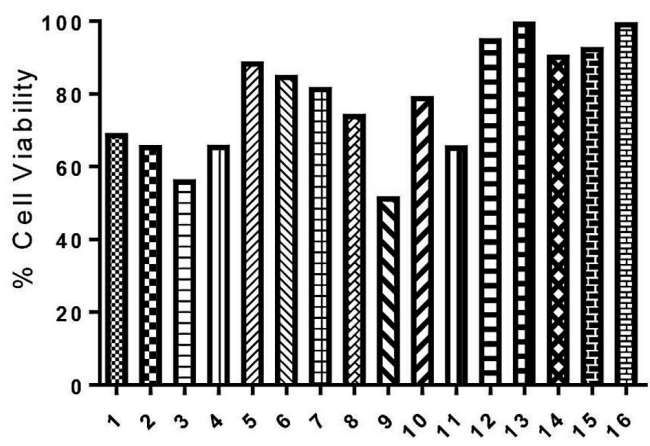

Patients

A M L 1

B

$C I=0.028$

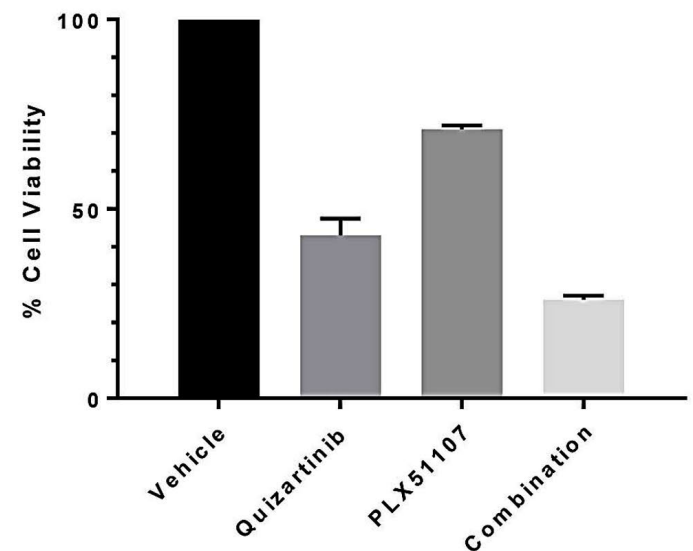

C Average Cell Viability CI $=0.064 ; n=10$

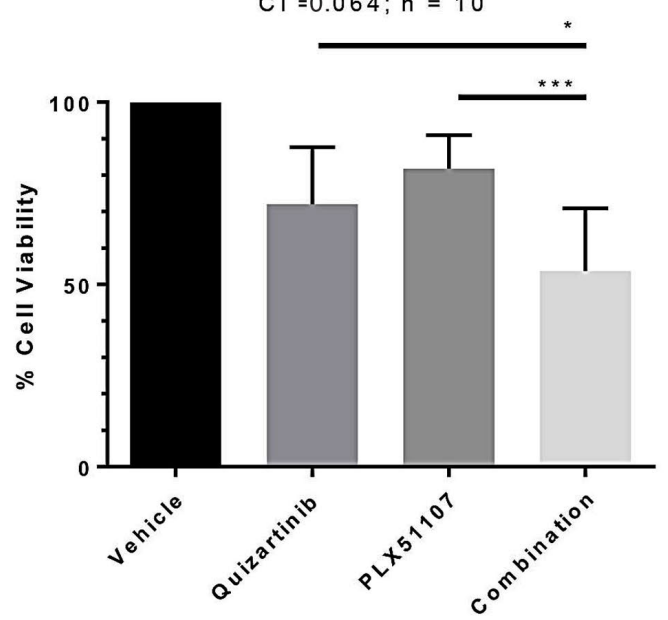

50 nM Quizartinib

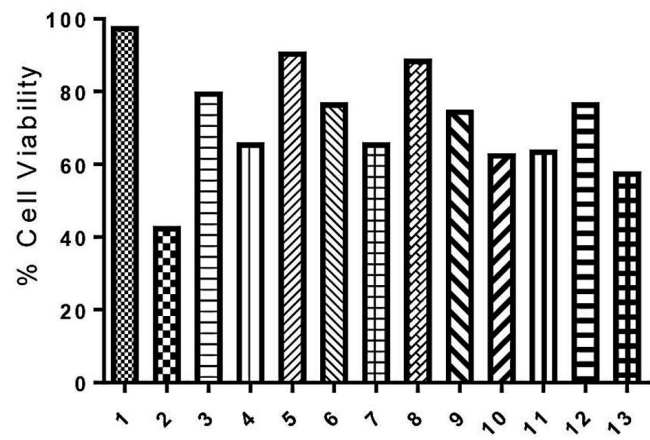

Patients

A M L 2

$C I=0.015$

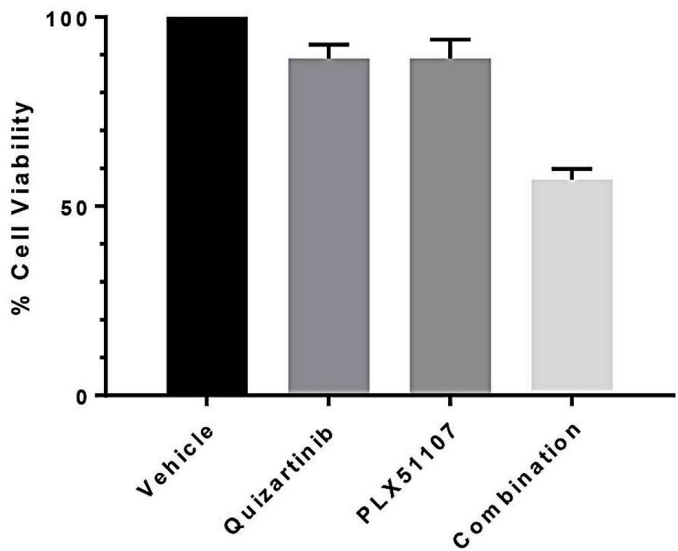

D

A M L 2

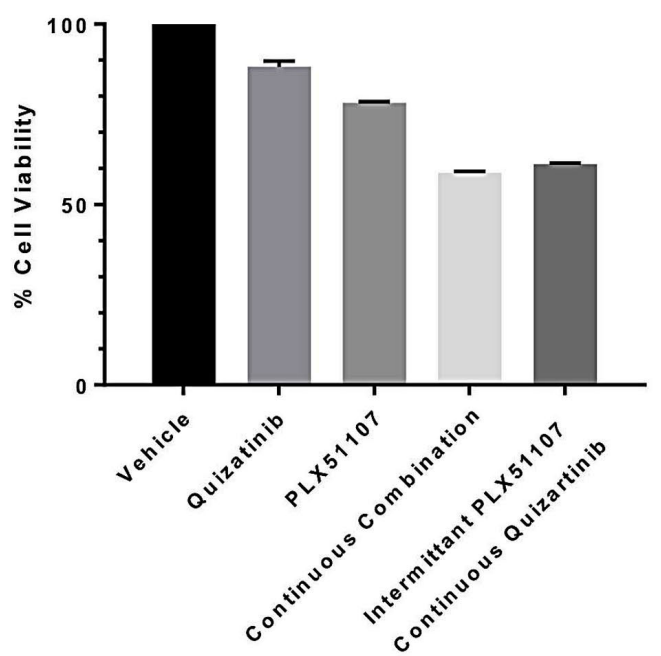

Figure 4. Synergistic cytotoxicity of PLX51107 and quizartinib in primary acute myeloid leukemia samples on bone marrow stroma. (A) Primary blasts from patients with relapsed or refractory FLT3-ITD AML were co-cultured with BM stromal cells in the presence of the clinically achievable doses of either 100 nM gilteritinib (left) or $50 \mathrm{nM}$ quizartinib (right) for 72 hours (h) and cell viability was assessed by a dimethyl-thiazole diphenol tetrazolium bromide (MTT) assay. Sample numbers in the gilteritinib series do not correspond to those in the quizartinib series, as they were assayed at different times. (B) Relapsed or refractory FLT3-ITD AML patients' samples were co-cultured with BM stroma and treated with $50 \mathrm{nM}$ quizartinib, $250 \mathrm{nM}$ PLX51107, or a combination (50 nM quizartinib and 250 nM PLX51107) for $72 \mathrm{~h}$ prior to assessment for cell viability by MTT. Overall, a total of ten AML samples were screened. AML1 and AML2 are two representative cases. Patients' characteristics for AML1 and AML2 are provided in Online Supplementary Table S1. (C) Averages of each condition from the ten primary samples are shown, with standard deviations represented by error bars. ${ }^{*} P<0.05$, $* * P<0.01$; two-tailed Student $t$-test. (D) Sample AML2 exposed to quizartinib and PLX51107 as in (B), but for this experiment, PLX51107 was washed out after $6 \mathrm{~h}$ exposure for each of the 3 days of exposure. 
A

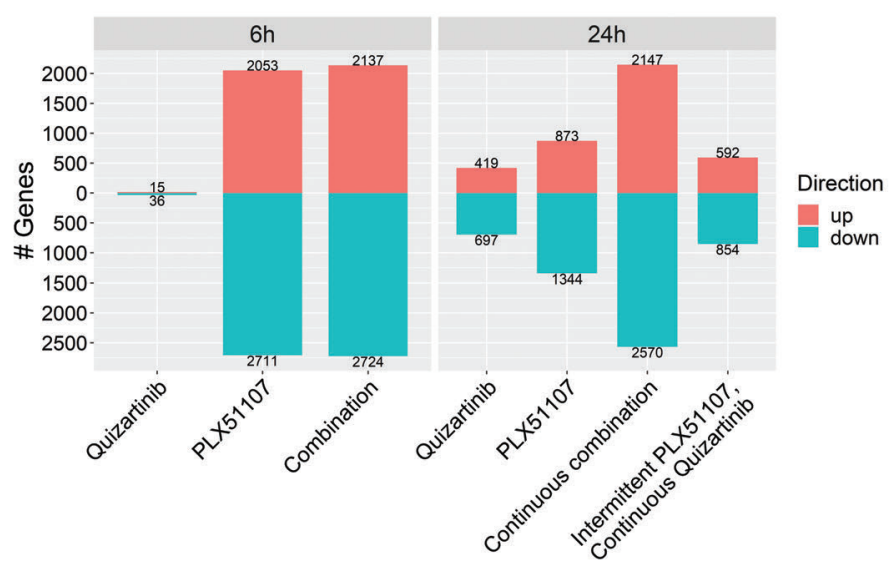

Figure 5. Legend on following page.

B

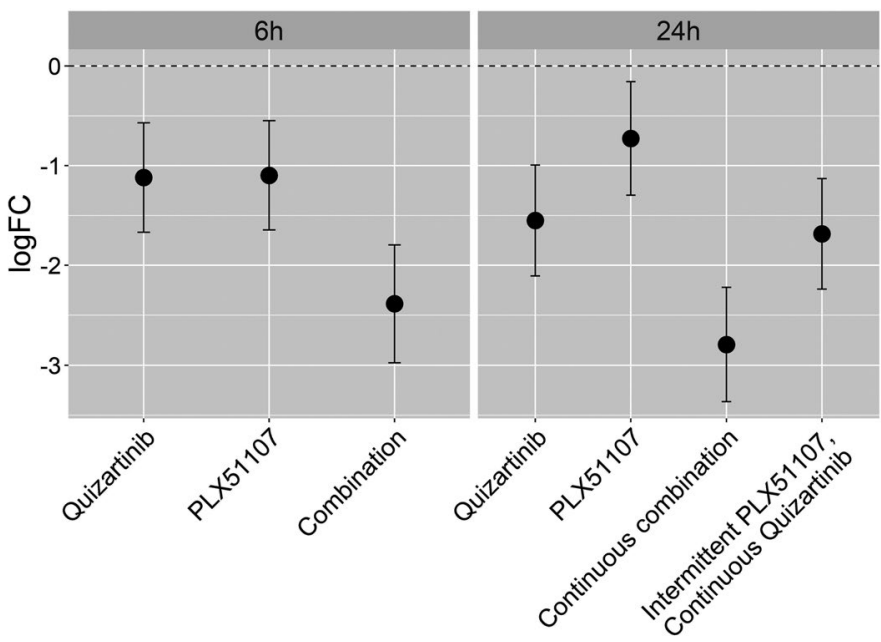

C

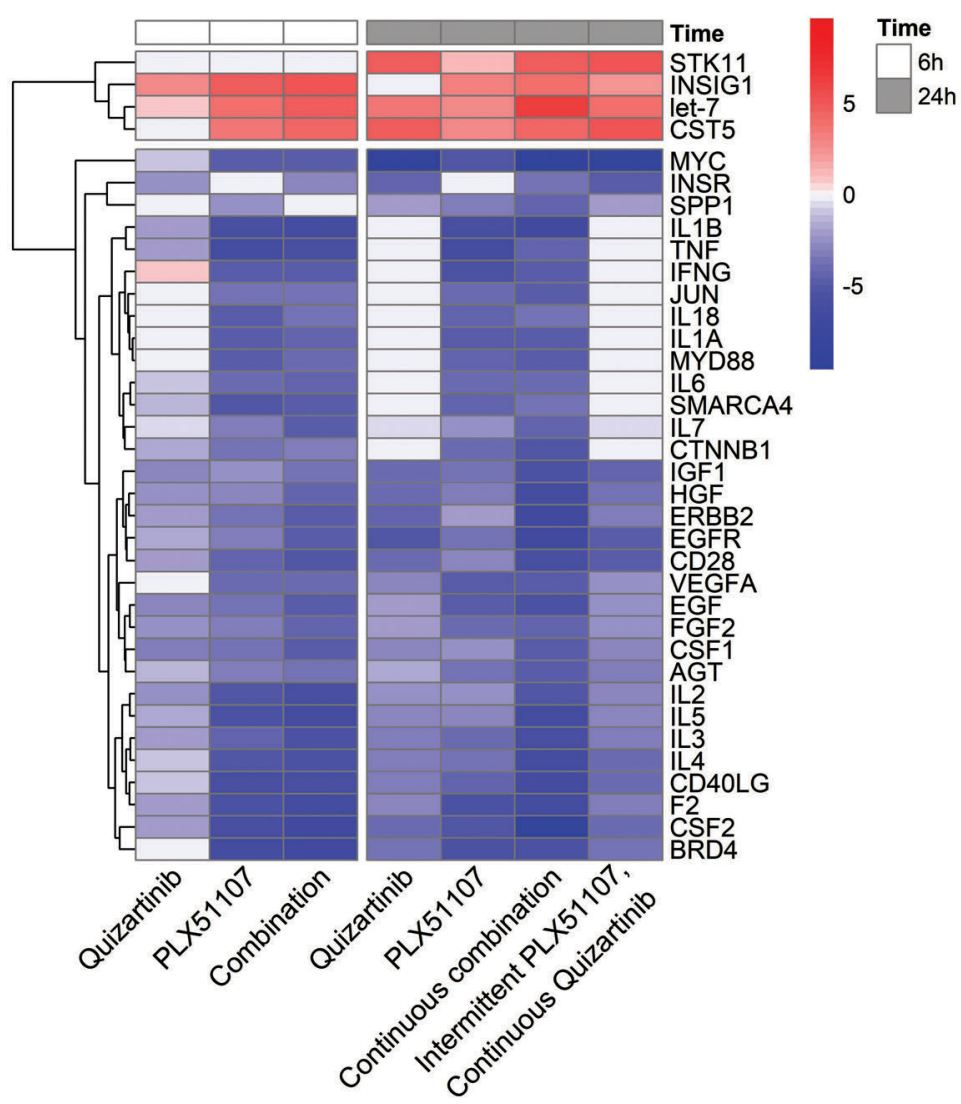


D

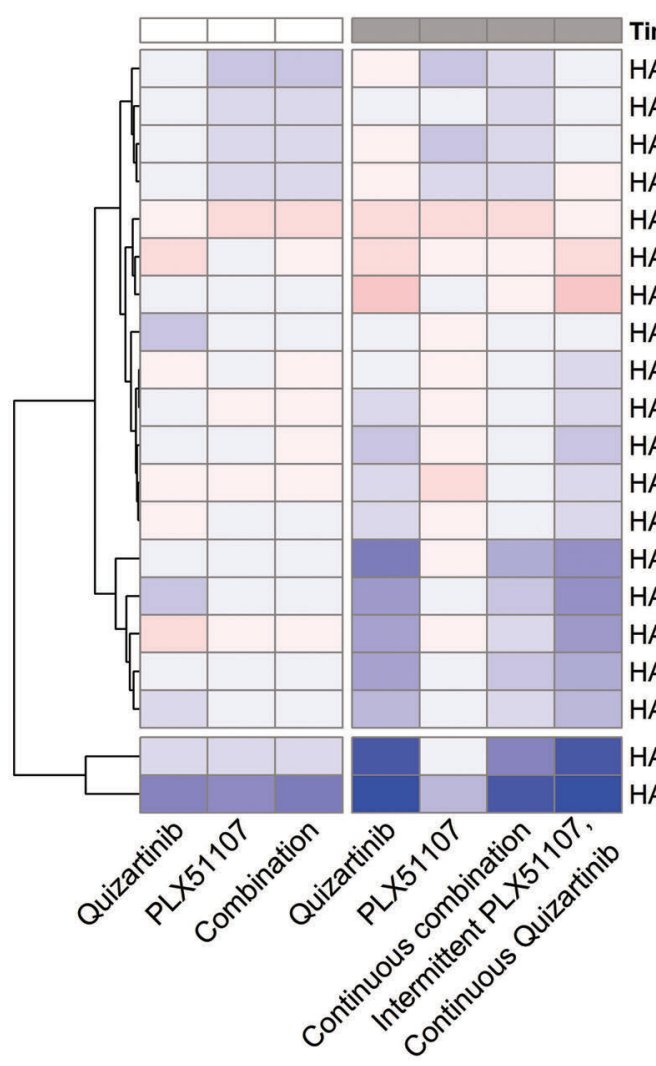

Time

HALLMARK_INFLAMMATORY_RESPONSE

HALLMARK_IL2_STAT5_SIGNALING

HALLMARK_ALLOGRAFT_REJECTION

HALLMARK_KRAS_SIGNALING_UP

HALLMARK_HEME_METABOLISM

HALLMARK_INTERFERON_ALPHA_RESPONSE

HALLMARK_UV_RESPONSE_DN

HALLMARK_CHOLESTEROL_HOMEOSTASIS

HALLMARK_ADIPOGENESIS

HALLMARK_UV_RESPONSE_UP

HALLMARK_GLYCOLYSIS

HALLMARK_DNA_REPAIR

HALLMARK_FATTY_ACID_METABOLISM

HALLMARK_E2F_TARGETS

HALLMARK_MTORC1_SIGNALING

HALLMARK_OXIDATIVE_PHOSPHORYLATION

HALLMARK_G2M_CHECKPOINT

HALLMARK_UNFOLDED_PROTEIN_RESPONSE

HALLMARK_MYC_TARGETS_V1

HALLMARK_MYC_TARGETS_V2

Figure 5. Gene expression. (A) Differentially expressed genes. Three different primary blasts from relapsed FLT3-ITD acute myeloid leukemia (AML) patients were treated with $50 \mathrm{nM}$ quizartinib, $250 \mathrm{nM}$ PLX51107, a continuous combination of both (50 nM quizartinib and 250 nM PLX51107), or intermittent dosing (6 hours [h]) of $250 \mathrm{nM}$ PLX51107 and continuous $50 \mathrm{nM}$ quizartinib. Cells were collected at both 6-h and 24-h time points for the transcriptome analysis. In each condition, the number of differentially expressed genes (averaged for the three samples) with false discovery rate (FDR) values $<0.05$ is shown. (B) Average expression of MYC for the three samples in (A) for each treatment condition compared to its time-matched baseline control. logFC values from the baseline are shown. (C) Heat map representation of the Ingenuity Pathway Analysis using the differentially expressed genes in each condition. Genes detected in (A) were used as input data. From the generated upstream factors, those that are not a single gene or miRNA were excluded. Upstream factors that had the highest activation z-scores in absolute value $>4.5$ were extracted. The color on the heat map indicates the activation z-scores and the heat map data are clustered by row. (D) Camera gene set enrichment analysis was performed on the three AML samples using MSigDB Hallmark gene sets. From the hallmark gene sets, those with -log ${ }_{10}$ (FDR) scores $>2$ were analyzed. Downregulated gene sets are indicated by negative values. Red: upregulated; blue: downregulated.

intermittent) exposure to PLX51107 in combination with continuous exposure to quizartinib yielded a pattern that was similar to that of quizartinib alone. Using both a simple iterative approach and a gene set enrichment analysis (GSEA), we identified MYC as one of the genes most affected by the combination of the two drugs (Figure $5 \mathrm{~B}$ ). We then used the upstream analysis function in the ingenuity pathway analysis (IPA) to analyze the changes induced by the drug exposure. The IPA confirmed that MYC-associated genes displayed the highest level of downregulation, even in cells that were intermittently exposed to BET inhibition (Figure 5C). Camera GSEA was also consistent with IPA upstream analysis (Figure 5D).

We confirmed that MYC RNA levels and protein were directly suppressed in these primary samples co-cultured on stroma using quantitative-PCR and immunoblotting (Figure 6A and $\mathrm{B}$ ). Six-hour exposure to BET inhibition resulted in augmented suppression of MYC transcript and protein, which resolved by $24 \mathrm{~h}$ (i.e., after $18 \mathrm{~h}$ of washout). The synergistic cytotoxic effects were apparently the result of suppressing MYC expression for just $6 \mathrm{~h}$.

The implications of the above findings are that combination therapy with approximately $120 \mathrm{mg}$ /day PLX51107 and $60 \mathrm{mg} /$ day quizartinib will be a more effective thera- py for relapsed FLT3-ITD AML than $60 \mathrm{mg} /$ day of quizartinib alone. However, quizartinib is myelosuppressive at doses higher than $60 \mathrm{mg} / \mathrm{day}^{23,26,27}$ and it is possible that the addition of PLX51107 will lead to greater marrow suppression. To investigate this possibility, we performed colony assays using normal BM-derived progenitor cells exposed to these two drugs. PLX51107 alone at concentrations below 1,000 $\mathrm{nM}$ had no discernible effect on erythroid or granulocyte-macrophage colony forming activity, even with 10-14 days of continuous exposure (Figure 7A). In the presence of $50 \mathrm{nM}$ quizartinib, $500 \mathrm{nM}$ PLX51107 did suppress colony formation (Figure 7B). However, in this experiment, the progenitor cells were exposed to BET inhibition continuously for 10-14 days, rather than the intermittent exposures used in the cytotoxicity assays. We predict that intermittent BET inhibition with continuous FLT3 inhibition would not cause intolerable myelosuppression in AML patients.

\section{Discussion}

The BM microenvironment protects blasts against toxins through diverse mechanisms, including expression of 
A C - M y c

Time (h): 0 6

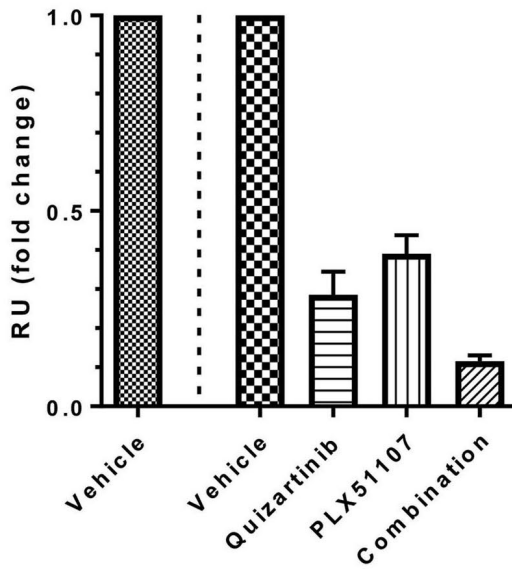

24

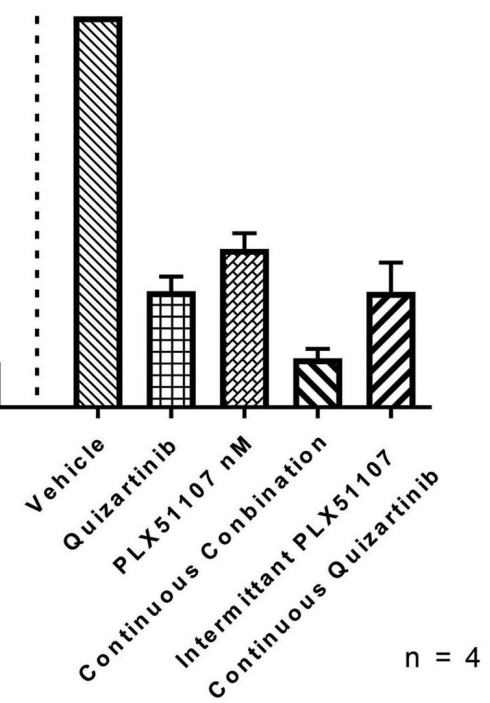

Figure 6. Primary samples co-cultured on stroma using quantitativepolymerase chain reaction (qPCR) and immunoblotting. (A) MYC expression in primary acute myeloid leukemia (AML) blasts by GPCR. Primary blasts from a patient with relapsed FLT3-ITD AML were co-cultured on stroma and treated under the specified condition. RNA was isolated from cells at 6 -hour $(\mathrm{h}$ ) and 24-h time points and analyzed as described in the Methods section. (B) MYC protein expression in primary AML blasts by immunoblotting. A primary FLT3-ITD AML sample (AML2, from Figure 4B) was treated with drug for $3 \mathrm{~h}$, lysed, and probed for MYC as described in the Methods section. $\beta$-actin was used as control for protein loading. Protein expression levels were quantified by densitometry.

B

AML2

c-Myc

Densitometry (\% of control):

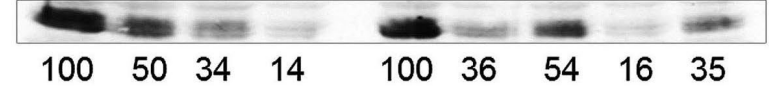

Actin

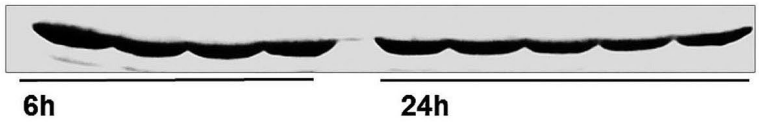

A

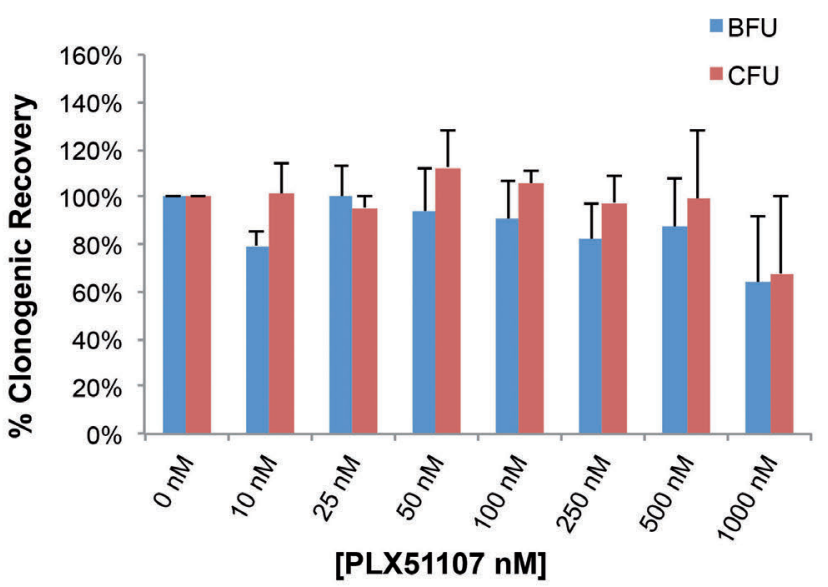

B

50nM Quizartinib + PLX51107

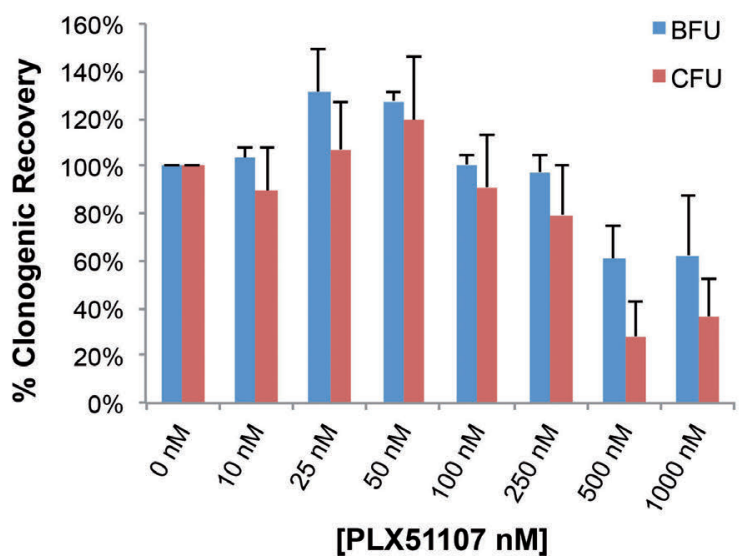

Figure 7. Colony forming cell assays. Colony assays were performed using normal bone marrow progenitor cells from healthy donors. Cells were cultured either with (A) PLX51107 alone or (B) with 50 nM quizartinib and increasing concentrations of PLX51107 for 10-14 days prior to assessment by microscopy. Averages of three individual experiments (three different donors) are shown; standard error of mean is shown as error bars. BFU: burst-forming unit; CFU: colony-forming unit. 
cytochrome P450 enzymes within stromal cells and the production of cytokines that activate pro-survival pathways within the leukemia cells. ${ }^{10,28}$ Overcoming the protective effect of marrow could lower disease burden in responding patients, and either directly prolong survival or allow more successful application of allogeneic transplantation.

We have turned to BET inhibitors to undermine the stroma-derived maintenance of pro-survival proteins in blasts within the marrow. BET inhibitors have attracted considerable interest as potential therapies for different diseases, but it seems unlikely that sustained suppression of BET protein activity would be tolerated in humans, a concern that has largely been borne out by early studies of first generation BET inhibitors. ${ }^{29,30}$ However, PLX51107 has a relatively short plasma half-life in human patients, and our ex vivo surrogate PIA assay for MYC indicates effective BET inhibition can be achieved with this drug even at modest daily doses. Using the MYC PIA assay, in combination with the FLT3 PIA assay, a plasma sample from a patient taking both a BET and FLT3 inhibitor can be assayed for each drug effect separately, using OCIAML3 cells for BET inhibition and MOLM-14 cells for the FLT3 inhibition. Even though intermittent, the BET inhibition induced by PLX51107 still results in synergistic cytotoxicity in combination with FLT3 inhibition against blasts on BM stromal cells. BET inhibition is expected to affect MYC and a myriad of other key genes, any number of which could contribute to the synergistic effect. ${ }^{18}$ Our use of MYC in the PIA assay is primarily a surrogate for BET activity, and, indeed, our data indicate that it is likely to be the most appropriate.

In designing a clinical protocol for this combination regimen, we will need to choose a clinically validated FLT3 inhibitor. Both gilteritinib and quizartinib prolong survival for relapsed FLT3-ITD AML, and both are synergistic with BET inhibition. FLT3-TKD mutations emerge in response to monotherapy with quizartinib, ${ }^{11}$ while RAS mutations emerge in response to gilteritinib therapy, ${ }^{31}$ so neither drug offers a particular advantage in this regard. Quizartinib is more myelosuppressive than gilteritinib, ${ }^{27}$ but, at least in our colony assays, the addition of BET inhibition does not seem to increase this. On the other hand, quizartinib, as a type II inhibitor, is more selective than gilteritinib, and its combination with another small molecule inhibitor may result in fewer off-target effects.

In summary, this work provides the scientific foundation for a clinical trial of a BET plus FLT3 inhibitor for the treatment of relapsed/refractory FLT3-ITD AML. The PIA assays for MYC and FLT3 can provide laboratory correlates to quantify in vivo inhibition of both targets in the dose escalation and expansion cohorts.

\section{Disclosures}

ML is a consultant to Daiichi-Sankyo, Astellas, Novartis, FujiFilm, Amgen, and Agios, and receives research funding from Astellas, FujiFilm, and Novartis; PS, BP, CZ, YM and GB are employees of Plexxikon, whose product, PLX51107, is a subject of this research; YH, MN and HS are employees of DaiichiSankyo, whose product, quizartinib, is a subject of this research; $L L, D H$ and TR have no conflict of interest to disclose.

\section{Contributions}

$L L$ performed experiments, analyzed data and wrote the manuscript; $Y H, P S, B P, C Z, Y M, M N, H S, D H, T R$ and $G R$ performed experiments, analyzed data and edited the manuscript; ML designed the study, analyzed data and wrote the manuscript.

\section{References}

1. Papaemmanuil E, Gerstung M, Bullinger L, et al. Genomic classification and prognosis in acute myeloid leukemia. N Engl J Med. 2016;374(23):2209-2221.

2. Levis M. FLT3 mutations in acute myeloid leukemia: what is the best approach in 2013? Hematology Am Soc Hematol Educ Program. 2013;2013:220-226.

3. Wattad M, Weber D, Dohner K, et al. Impact of salvage regimens on response and overall survival in acute myeloid leukemia with induction failure. Leukemia. 2017; 31(6):1306-1313.

4. Daver N, Schlenk RF, Russell NH, Levis MJ. Targeting FLT3 mutations in AML: review of current knowledge and evidence. Leukemia. 2019;33(2):299-312.

5. Chevallier P, Labopin M, Turlure P, et al. A new Leukemia Prognostic Scoring System for refractory/relapsed adult acute myelogeneous leukaemia patients: a GOELAMS study. Leukemia. 2011;25(6):939-944.

6. Ravandi F, Kantariian H, Faderl S, et al. Outcome of patients with FLT3-mutated acute myeloid leukemia in first relapse. Leuk Res. 2010;34(6):752-756.

7. Cortes J, Perl AE, Dohner $\mathrm{H}$, et al. Quizartinib, an FLT3 inhibitor, as monotherapy in patients with relapsed or refractory acute myeloid leukaemia: an open-label, multicentre, single-arm, phase 2 trial. Lancet Oncol. 2018;19(7):889-903
8. Perl AE, Altman JK, Cortes J, et al. Selective inhibition of FLT3 by gilteritinib in relapsed or refractory acute myeloid leukaemia: a multicentre, first-in-human, open-label, phase 1-2 study. Lancet Oncol. 2017; 18(8):1061-1075.

9. Sexauer A, Perl A, Yang X, et al. Terminal myeloid differentiation in vivo is induced by FLT3 inhibition in FLT3/ITD AML. Blood. 2012;120(20):4205-4214.

10. Yang X, Sexauer A, Levis M. Bone marrow stroma-mediated resistance to FLT3 inhibitors in FLT3-ITD AML is mediated by persistent activation of extracellular regulated kinase. Br J Haematol. 2014;164(1):61-72

11. Smith CC, Wang O, Chin CS, et al Validation of ITD mutations in FLT3 as a therapeutic target in human acute myeloid leukaemia. Nature. 2012;485(7397):260-263

12. Piloto $O$, Wright M, Brown P, Kim KT, Levis M, Small D. Prolonged exposure to FLT3 inhibitors leads to resistance via activation of parallel signaling pathways. Blood. 2007; 109(4):1643-1652

13. Zhang $H$, Savage S, Schultz AR, et al Clinical resistance to crenolanib in acute myeloid leukemia due to diverse molecular mechanisms. Nat Commun. 2019;10(1):244

14. Parmar A, Marz S, Rushton S, et al. Stromal niche cells protect early leukemic FLT3ITD+ progenitor cells against first-generation FLT3 tyrosine kinase inhibitors. Cancer Res. 2011;71(13):4696-4706.

15. Traer E, Martinez J, Javidi-Sharifi N, et al.
FGF2 from marrow microenvironment promotes resistance to FLT3 inhibitors in acute myeloid leukemia. Cancer Res. 2016;76(22): 6471-6482.

16. Winter GE, Mayer A, Buckley DL, et al. BET bromodomain proteins function as master transcription elongation factors independent of CDK9 recruitment. Mol Cell. 2017; 67(1):5-18.

17. Shi J, Vakoc CR. The mechanisms behind the therapeutic activity of BET bromodomain inhibition. Mol Cell. 2014;54(5):728-736.

18. Delmore JE, Issa GC, Lemieux ME, et al BET bromodomain inhibition as a therapeutic strategy to target c-Myc. Cell. 2011; 146(6):904-917.

19. Fiskus W, Sharma S, Oi J, et al. BET protein antagonist JQ1 is synergistically lethal with FLT3 tyrosine kinase inhibitor (TKI) and overcomes resistance to FLT3-TKI in AML cells expressing FLT-ITD. Mol Cancer Ther. 2014;13(10):2315-2327.

20. Ozer HG, El-Gamal D, Powell B, et al. BRD4 profiling identifies critical chronic lymphocytic leukemia oncogenic circuits and reveals sensitivity to PLX51107, a novel structurally distinct BET inhibitor. Cancer Discov. 2018;8(4):458-477

21. Pratz KW, Sato T, Murphy KM, Stine A, Rajkhowa T, Levis M. FLT3-mutant allelic burden and clinical status are predictive of response to FLT3 inhibitors in AML. Blood. 2010;115(7):1425-1432.

22. Levis M, Brown P, Smith BD, et al. Plasma 
inhibitory activity (PIA): a pharmacodynamic assay reveals insights into the basis for cytotoxic response to FLT3 inhibitors. Blood. 2006;108(10):3477-3483.

23. Galanis A, Ma H, Rajkhowa $\mathrm{T}$, et al. Crenolanib is a potent inhibitor of FLT3 with activity against resistance-conferring point mutants. Blood. 2014;123(1):94-100.

24. Cortes JE, Kantarjian H, Foran JM, et al. Phase I study of quizartinib administered daily to patients with relapsed or refractory acute myeloid leukemia irrespective of FMSlike tyrosine kinase 3-internal tandem duplication status. J Clin Oncol. 2013; 31(29):3681-3687.

25. Levis MJ, Cortes JE, Gammon GM, Trone D, Kang D, Li J. Laboratory and clinical investi- gations to identify the optimal dosing strategy for quizartinib (AC220) monotherapy in FLT3-ITD-positive (+) relapsed/refractory $(\mathrm{R} / \mathrm{R})$ acute myeloid leukemia (AML). Blood. 2016;128(22):4042-4042

26. Galanis A, Levis M. Inhibition of c-Kit by tyrosine kinase inhibitors. Haematologica. 2015;100(3):e77-79.

27. Lee LY, Hernandez D, Rajkhowa T, et al Preclinical studies of gilteritinib, a next-generation FLT3 inhibitor. Blood. 2017; 129(2):257-260

28. Chang YT, Hernandez D, Alonso $S$, et al. Role of CYP3A4 in bone marrow microenvironment-mediated protection of FLT3/ITD AML from tyrosine kinase inhibitors. Blood Adv. 2019;3(6):908-916.
29. Berthon C, Raffoux E, Thomas X, et al. Bromodomain inhibitor OTX015 in patients with acute leukaemia: a dose-escalation, phase 1 study. Lancet Haematol. 2016; 3(4):e186-195.

30. Amorim S, Stathis A, Gleeson M, et al Bromodomain inhibitor OTX015 in patients with lymphoma or multiple myeloma: a dose-escalation, open-label, pharmacokinetic, phase 1 study. Lancet Haematol. 2016; 3(4):e196-204

31. McMahon CM, Ferng T, Canaani J, et al Clonal selection with Ras pathway activation mediates secondary clinical resistance to selective FLT3 inhibition in acute myeloid leukemia. Cancer Discov. 2019;9(8):10501063. 